\title{
Downregulation of Foxc2 enhances apoptosis induced by 5-fluorouracil through activation of MAPK and AKT pathways in colorectal cancer
}

\author{
CHAO YANG $^{1}$, XIAOXIAN CUI $^{1}$, XIAOQIN DAI $^{1}$ and WENTING LIAO ${ }^{1,2}$ \\ ${ }^{1}$ Department of Pathology, School of Basic Medical Science; ${ }^{2}$ Department of Pathology, \\ Nanfang Hospital, Southern Medical University, Guangzhou, Guangdong 510515, P.R. China
}

Received February 6, 2015; Accepted December 18, 2015

DOI: $10.3892 / 01.2016 .4097$

\begin{abstract}
The chemotherapy drug 5-fluorouracil (5-FU) is fundamental for the treatment of colorectal cancer (CRC); however, drug resistance to 5-FU may occasionally occur. Abnormal expression of Forkhead box C2 gene (Foxc2) has been identified in several human cancers, but the role of Foxc2 in the progression of CRC remains unclear. The present study established a stable Foxc2-short hairpin (sh)RNA cell line, which was confirmed by western blot analysis and quantitative polymerase chain reaction. The Foxc2-shRNA cells were treated with 5-FU and the cell viability was determined by an MTT assay. Western blot analysis was performed to investigate the signaling pathway involved in 5-FU treatment. The present study identified that $5-\mathrm{FU}$ increased the percentage of apoptotic CRC cells among the Foxc2/RNA interference-transfected cells compared with cells transfected with an empty vector. Therefore, the downregulation of Foxc2, induced by 5-FU, may enhance apoptosis by the downregulation of apoptotic factors, including B cell lymphoma-2 and pro-caspase-3, in Foxc2-shRNA CRC cells. Furthermore, the mitogen-activated protein kinase (MAPK) and phosphatidylinositide 3-kinases/protein kinase B (PI3K/AKT) pathways were essential for the sensitization effect of Foxc2 to 5-FU treatment. Overall, these findings reveal the mechanisms behind Foxc2 depletion and 5-FU treatment of CRC and suggest that Foxc2 enhances resistance to apoptosis, induced by $5-\mathrm{FU}$, through the activation of MAPK and P13K/AKT pathways, and may serve as a valuable clinical prognostic marker for CRC.
\end{abstract}

Correspondence to: Dr Wenting Liao, Department of Pathology, Nanfang Hospital, Southern Medical University, 1838 North Avenue, Guangzhou, Guangdong 510515, P.R. China

E-mail: liaowt2002@gmail.com

Key words: colorectal cancer, Foxc2, apoptosis, MAPK, AKT

\section{Introduction}

Colorectal cancer (CRC) is the third leading cause of cancer-associated mortality and morbidity in the USA, accounting for $9 \%$ of novel cases and mortalities that affect men and women (1). At present, the available diagnostic methods for CRC, including colonoscopy and fecal occult-blood test, remain unsatisfactory (2), and common premalignant lesions, such as sessile serrated adenomas, are extremely challenging to identify. The 5-year survival rate for patients with early stage CRC is $\sim 80 \%$, following radical surgery (2). In addition, during the process of CRC cell apoptosis induced by chemotherapy, tolerant cells often appear and escape treatment. Therefore, novel effective targets for anti-CRC treatment are required.

The forkhead box C2 (Foxc2) gene was first identified in mice during embryogenesis (3). The gene was then observed at high expression levels in a variety of malignancies, including esophageal (4), breast (5) and prostate (6) cancers. In addition, there is evidence that suggests that Foxc2 is associated with the regulation of cell proliferation, the development of angiogenesis (7) and the metastasis of human tumors (8-10). Previous studies have established that Foxc2 plays a predominant role in the modulation of cancer cells, which resist apoptosis-based tumor surveillance and treatments (11). Petrova et al reported that Foxc2 is highly expressed in developing lymphatic vessels and lymphatic valves (12). However, the functional role of Foxc2 has not yet been investigated under physiological and pathological conditions.

A preparatory study revealed that Foxc2 expression is significantly associated with the progression of CRC in vitro and in vivo, using reverse transcription-polymerase chain reaction (RT-PCR) and western blot analysis. This result is also demonstrated in CRC samples in paraffin-embedded tissues (13). Foxc2 was expressed in CRC patients and upregulated in the majority of these patients. Furthermore, the downregulation of Foxc2 lead to the apoptosis of cancer cells. Based on these results, the present study hypothesizes that the ablation of Foxc2 expression may lead human cancer cells, including CRC cells, to become sensitive to chemotherapy.

The present study, to the best of our knowledge, is the first to demonstrate that the morphological alterations observed 
in 5-fluorouracil (5-FU)-induced apoptosis are paralleled by Foxc2 deregulation. These results may have implications for the design of chemotherapy treatment; the combination of 5-FU treatment and Foxc2 depletion may lead to an improved treatment strategy for CRC patients.

\section{Materials and methods}

Cell lines. The study was approved by the Ethics Committee of Southern Medical University (Guangzhou, Guangdong, China). The human colon carcinoma HCT116 cell line was obtained from the American Type Culture Collection (Manassas, VA, USA). These cells were grown in Gibco RPMI-1640 media (Thermo Fisher Scientific, Inc., Waltham, MA, USA) and supplemented with $10 \%$ fetal bovine serum (GE Healthcare, Life Sciences, Chalfont, UK) and 100 units Invitrogen penicillin-streptomycin (Thermo Fisher Scientific, Inc.) at $37^{\circ} \mathrm{C}$, with a $5 \% \mathrm{CO}_{2}$ atmosphere in a humidified incubator.

Retroviral infection and reverse transcription. The Foxc2 expression construct was generated by cloning PCR-amplified, full-length or deletion mutant human Foxc2 cDNA into a pBabe plasmid (Addgene, Inc., Cambridge, MA, USA). RNA was extracted from the HCT116 cells using Trizol reagent (Thermo Fisher Scientific, Inc.), as previously described (14). Foxc2 cDNA was obtained using reverse transcription, which was performed using the Invitrogen SuperScript ${ }^{\mathrm{TM}}$ First-Strand Synthesis System for RT-PCR (Thermo Fisher Scientific, Inc.), according to the manufacturer's protocol. The human shRNA sequence, as described by Mani et al (15), was 5'-CCACAC GTTTGCAACCCAA-3'. To knockdown Foxc2, the sequence was cloned into a pSUPER.retro.neo vector (Oligoengine, Seattle, WA, USA). Retroviral production and infection were performed, as previously described (16).

Quantitative PCR (qPCR) and western blot analysis. qPCR and western blot analysis were performed to confirm the successful infection of the CRC cells. qPCR was performed using the Applied Biosystems ABI PRISM ${ }^{\circledR} 7500$ Sequence Detection System (Thermo Fisher Scientific Inc.) and the $\mathrm{iQ}^{\mathrm{TM}}$ SYBR ${ }^{\circledR}$ Green Supermix (Bio-Rad Laboratories, Inc., Hercules, CA, USA), which contained 5 ng cDNA and 10 pM of each primer. The reference gene glyceraldehyde-3-phosphate dehydrogenase (GAPDH) was used as an internal quantitative control. The methods used were as previously described (17). The thermal cycling conditions for the qPCR were as follows: Denaturation at $95^{\circ} \mathrm{C}$ for $5 \mathrm{~min} ; 40$ cycles of denaturation at $95^{\circ} \mathrm{C}$ for $15 \mathrm{sec}$; annealing at $56.4^{\circ} \mathrm{C}$ for $20 \mathrm{sec}$; and extension at $72^{\circ} \mathrm{C}$ for $20 \mathrm{sec}$. Primers were designed using Applied BioSystems Primer Express version 2.0 software (Thermo Fisher Scientific, Inc.), as follows: Foxc2 sense, 5'-CTACAGCTACATCGCGCT CATCA-3' and antisense, 5'-ACTGGTAGATGCCGTTCA AGGTG-3'; Bmi-1 sense, 5'-CTGGTTGCCCATTGA CAGC-3' and antisense, 5'-CAGAAAATGAATGCG AGCCA-3'; and GAPDH sense, 5'-GACTCATGACCACAG TCCATGC-3' and antisense, 5'-AGAGGCAGGGATGAT GTTCTG-3'. qPCR was performed using the Applied BioSystems SYBR Green I Nucleic Acid Gel Stain (Thermo
Fisher Scientific, Inc.). The data was normalized to the geometric mean of the reference gene GAPDH, and calculated using the $2^{-\Delta \Delta \mathrm{Cq}}$ method (18). For western blot analysis, an anti-Foxc2 rabbit anti-mouse monoclonal antibody (dilution, 1:1000; catalog no. A302-383A Bethyl Laboratories, Inc., Montgomery, TX, USA) was used.

Assessment of sensitivity to 5-FU using a 3-(4,5-dimethylthiazol-2-yl)-2,5-diphenyltetrazolium bromide (MTT) assay. Following synchronization for $24 \mathrm{~h}$ in a serum-free medium, a total of $5 \times 10^{3}$ cells were seeded in 6-well plates and treated with $0.5,1,2$ or 4 mg/1 5-FU (Sigma-Aldrich, Saint Louis, MO, USA) for 48 and 72 h. Cell viability was determined by an MTT assay. In brief, the cells were seeded on a 96-well plate $\left(10^{3}\right.$ cells/well), and on the following day $20 \mu \mathrm{l} /$ well of sterile MTT dye ( $5 \mathrm{mg} / \mathrm{ml}$; Sigma-Aldrich) was added to the cells. Following $4 \mathrm{~h}$ incubation at $37^{\circ} \mathrm{C}$, the medium was discarded and $150 \mu \mathrm{l} /$ well of dimethyl sulfoxide (Sigma-Aldrich) was added. The absorbance was measured at $570 \mathrm{~nm}$. The experiments were repeated 3 times.

Detecting apoptotic and MAPK and PI3K/AKT pathway markers in CRC cells by western blot analysis. Western blot analysis was used to observe the apoptosis of the cells, induced by 5 -FU and the depletion of Foxc2, and to detect mitogen-activated protein kinase (MAPK) and phosphatidylinositide 3-kinases/protein kinase B (PI3K/AKT) pathways. The following primary rabbit anti-mouse monoclonal antibodies were used, at a dilution of 1:1,000 unless otherwise stated: Anti-extracellular-signal-regulated kinases (ERK; catalog no. BS3627), anti-phosphorylated (p)-ERK (catalog no. BS4621), anti-c-Jun N-terminal kinases (JNK; catalog no. BS1544), anti-p-JNK (catalog no. BS4322), anti-p-P38 (catalog no. BS4766), anti-AKT (catalog no. BS2987), anti-p-AKT (catalog no. BS4007), anti-pro-caspase-3 (catalog no. BS1518), anti-cleaved-caspase-3 (catalog no. BS9661S), anti-B cell lymphoma (Bcl)-2 (catalog no. BS1511) and anti-Bcl-2-associated X protein (Bax; dilution, 1:100; catalog no. BS2538) (Bioworld Technology, Inc., St. Louis Park, MN, USA). Bcl-2, Bax and pro-caspase-3 are markers for apoptosis, while the other antigens are markers for the MAPK and PI3K/AKT pathways. Western blot analysis was performed as previously described (17). Briefly, equal amounts of protein were separated by electrophoresis on a $10 \%$ SDS-PAGE gel and electrotransferred from the gel to a nitrocellulose membrane (Merck \& Co., Inc., Whitehouse Station, NJ, USA). Following blocking with 5\% milk solution (Yili Group Co., Ltd., Neimenggu, China) for $2 \mathrm{~h}$ at room temperature, the membranes were incubated with the primary antibodies at $4^{\circ} \mathrm{C}$ overnight. The membranes were washed three times in Tris-buffered saline with Tween-20 (TBS-T; Merck \& Co., Inc.). Anti- $\alpha$-tubulin rabbit anti-mouse monoclonal antibody (dilution, 1:2,000; catalog no. KM9007; Tianjin Sungene Biotech Co., Ltd., Tianjin, China) was used as an internal loading control. Following washing with TBS-T, the nitrocellulose membrane was incubated with a secondary antibody against rabbit immunoglobulin (Ig)G or mouse IgG. The membrane was examined using an FluorChem FC2 Imaging System (Alpha Innotech, San Leandro, CA, USA), according to the manufacturer's protocol. 
Table I. Data obtained from the MMT assay, which examined the effect of 5-fluorouracil on the survival of Foxc2 knockdown cells.

\begin{tabular}{lcccc}
\hline & \multicolumn{2}{c}{ HCT116-vector, control } & \multicolumn{2}{c}{ HCT116-Foxc2/RNAi cells } \\
\cline { 2 - 3 } Variable & Mean & SD & Mean & \\
\hline Data set 1 & & & 0.133288900 & 0.034042870 \\
0.5 & 0.149116600 & 0.096946720 & 0.404865100 & 0.025722880 \\
1 & 0.277738500 & 0.062077910 & 0.377874000 & 0.067800570 \\
2 & 0.327444000 & 0.062361180 & 0.486171300 & 0.033669970 \\
4 & 0.407302700 & 0.126097800 & & 0.054933290 \\
Data set 2 & & & 0.473311200 & 0.021126600 \\
0.5 & 0.420335700 & 0.059749480 & 0.637435200 & 0.014314910 \\
1 & 0.583677900 & 0.074168170 & 0.811879100 & 0.009069179 \\
2 & 0.620165400 & 0.042991280 & 0.068660390 & \\
\hline
\end{tabular}

Foxc2, forkhead box C2 gene; HCT116, human colon cancer HCT116 cell line; RNAi, RNA interference; SD, standard deviation.

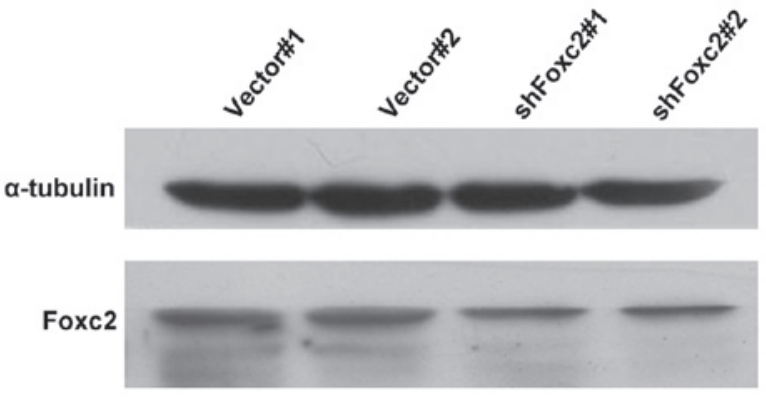

Figure 1. Stable cell lines of Foxc2 shRNA were established. The protein expression levels of Foxc2 were determined by western blot analysis. Total protein was electrophoresed and separated on a 10\% SDS-PAGE gel. Foxc2, forkhead box C2 gene; vector\#1, vector cells without 5-fluorouracil treatment; vector\#2, vector cells with $2 \mathrm{mg} / 1$ 5-fluorouracil; shFoxc2\#1, Foxc2 short hairpin RNA stable cells lines without 5-fluorouracil treatment; shFoxc2\#2, Foxc2 short hairpin RNA stable cells lines with $2 \mathrm{mg} / 1$ 5-fluorouracil.

Statistical analysis. Statistical analyses were performed using SPSS version 13.0 software (SPSS, Inc., Chicago, IL, USA). Comparisons between groups for statistical significance were performed using a two-tailed paired Student's $t$ test. $\mathrm{P}<0.05$ was considered to indicated a statistically significant difference.

\section{Results}

Establishment of Foxc2 small hairpin (sh) RNA stable cell lines. Foxc2-shRNA stable cell lines were established to study the role of Foxc2 in CRC cell survival. Western blot analysis and RT-qPCR revealed that protein and mRNA expression of Foxc2 was markedly downregulated in HCT116-shFoxc2 cells, in contrast to those in HCT116-vector cells (Figs. 1 and 2).

Ablation of Foxc2 expression leads to human colon cancer HCT116 cell lines becoming sensitized to 5-FU. An MTT assay was performed to examine the effect of 5-FU on the survival rate of Foxc2-knockdown cells. The cells were treated with 5-FU for 48 and $72 \mathrm{~h}$, and it was demonstrated that downregulation of Foxc2 significantly decreased the growth rate of HCT116 cells compared with vector cells. In detail, the present study identified that the viability of the HCT116-Foxc2/RNA interference cells were decreased compared with HCT116-vector cells (control). Following treatment with 5-FU for $48 \mathrm{~h}$, the half maximal inhibitory concentration $\left(\mathrm{IC}_{50}\right)$ of 5 -FU in the HCT116-vector and HCT116-shFoxc2 cells were $6.3919 \mathrm{mg} / 1$ and $5.2965 \mathrm{mg} / 1$ (P>0.05), respectively. Following treatment for $72 \mathrm{~h}$, the $\mathrm{IC}_{50}$ decreased to 1.8839 and $1.1238 \mathrm{mg} / \mathrm{l}$, respectively $(\mathrm{P}<0.05$; Fig. 3; Table I). The results suggest that following a depletion of Foxc2 all the cells become sensitized to 5-FU, particularly following a 72-h treatment time.

Foxc2 aids cells in resisting apoptosis and regulating the expression levels of Bcl-2, Bax and caspase-3. Due to the unsatisfactory clinical therapies available, as a result of the resistance of cancer cells to apoptosis, the present study investigated whether Foxc2 enhanced the anti-apoptotic activity of CRC cells. To investigate the mechanism by which apoptosis is upregulated when treated with 5-FU and a knockdown of Foxc2, the expression levels of the apoptosis regulators Bcl-2, Bax, pro-caspase-3 and cleaved-caspase-3 were observed in HCT116-shFoxc2 and HCT116-vector cells. Western blot analysis revealed a downregulation of Bcl-2 and pro-caspase-3 in Foxc2-knockdown cells, while Bax and cleaved-caspase-3 were upregulated (Fig. 4).

MAPK and PI3K/AKT pathways are essential for the sensitization effect of Foxc 2 to $5-F U$ treatment. The PI3K/AKT signaling pathway is an important survival pathway in numerous cellular systems and the activation of this pathway is required to prevent cell apoptosis $(19,20)$. To investigate whether the depletion of Foxc 2 enhances the apoptosis of cells through the PI3K/AKT pathways, the present study analyzed p-AKT and total AKT levels. As revealed in Fig. 5, p-AKT was decreased in HCT116-shFoxc2 cells, but the total AKT levels were not 

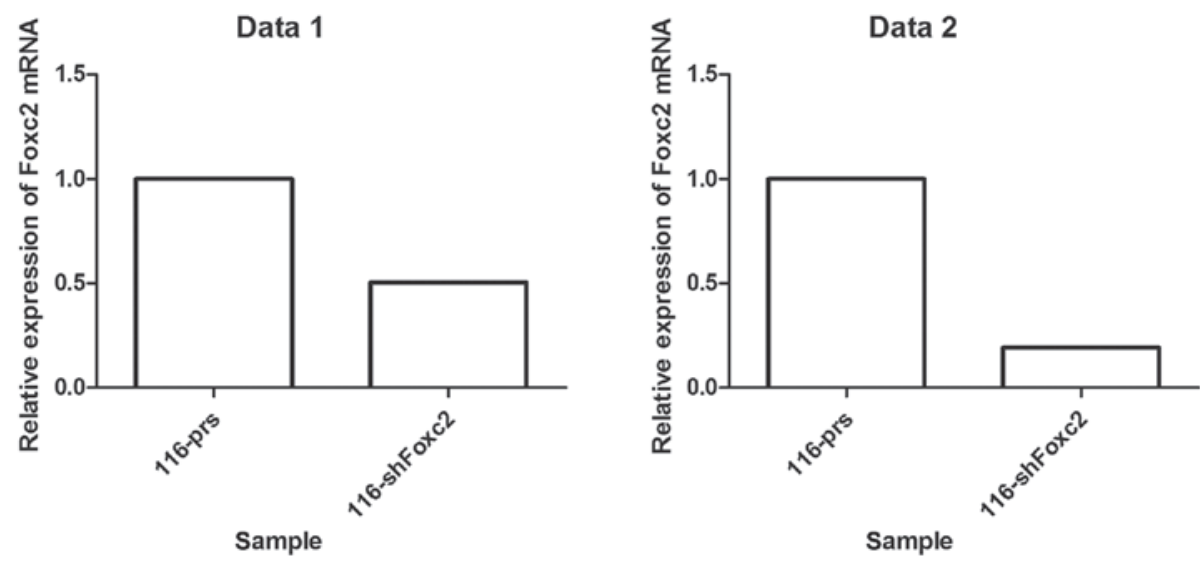

Figure 2. Reverse transcription-quantitiative polymerase chain reaction was performed twice, data sets 1 and 2, to confirm that stable cell lines of Foxc2 shRNA had been established. The percentages of HCT-116shFoxc2 are 0.5039 and $0.1928 \%$ in data sets 1 and 2 , respectively. Foxc2, forkhead box C2 gene, 116-prs, human colon cancer HCT-116 cell line; 116-shFoxc2, human colon cancer HCT-116 cell line with short hairpin Foxc2 RNA.
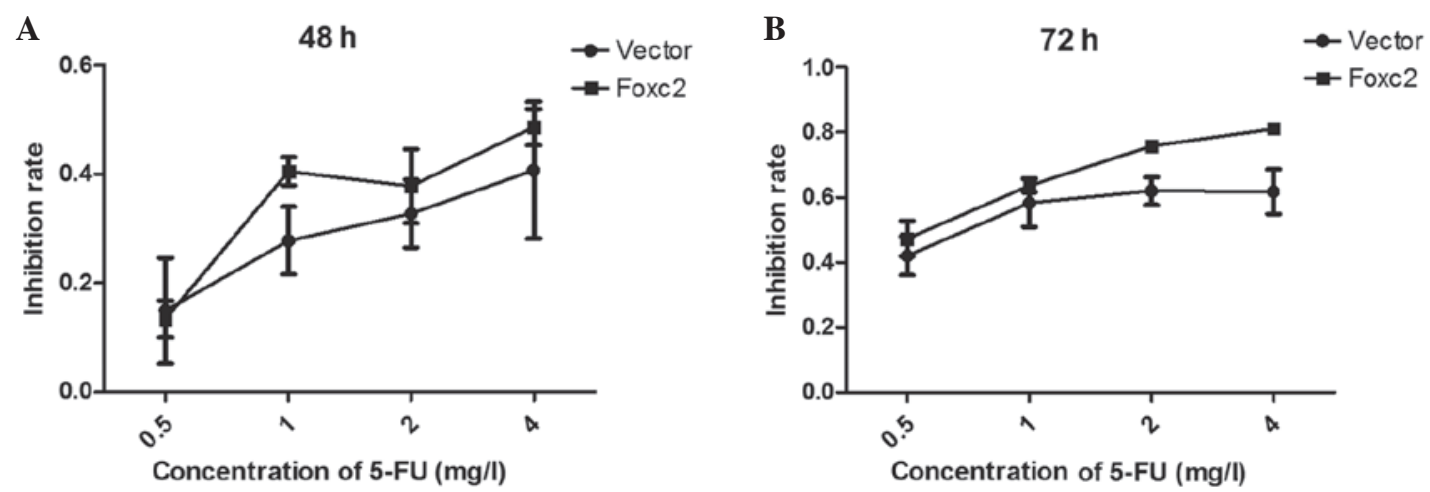

Figure 3. The effects of Foxc2 downregulation on the sensitivity of the human colon cancer HCT116 cell line to 5-FU treatment. HCT116-vector and HCT116-Foxc2/RNA interference cells were treated with increasing concentrations of 5-FU for (A) $48 \mathrm{~h}$ and (B) $72 \mathrm{~h}$. Cell viability was determined by an MTT assay. Foxc2, forkhead box C2 gene; 5-FU, 5-fluorouracil.

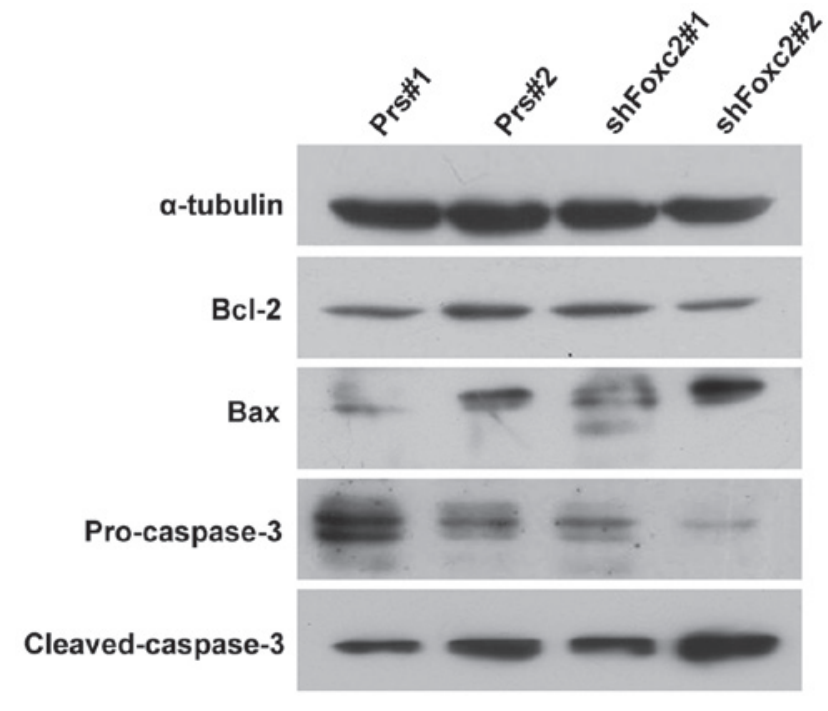

Figure 4. The effect of a knockdown of Foxc2. Cells were treated with or without $2 \mathrm{mg} / 15$-FU for $72 \mathrm{~h}$. The protein levels of Bcl-2, Bax, pro-caspase-3 and cleaved-caspase- 3 were determined by western blot analysis. The total protein was electrophoresed and separated on a 10\% SDS-PAGE gel. Foxc2, forkhead box C2 gene; 5-FU, 5-fluorouracil; Bcl-2, B cell lymphoma 2; Prs\#1, human colon cancer HCT116 cells without treatment of 5-FU; Prs\#2, human colon cancer HCT116 cells with 2 mg/1 5-FU; shFoxc2\#1, Foxc2 short hairpin RNA stable cell lines without treatment of 5-FU; shFoxc2\#2, Foxc2 short hairpin RNA stable cell lines with $2 \mathrm{mg} / 1$ 5-FU. altered. The result confirms that the PI3K/AKT pathway plays an important role in the sensitization effect of Foxc2 to 5-FU treatment.

In addition, in the MAPK pathway, the present study observed that although the total levels of ERK and JNK were not significantly altered subsequent to treatment with $5-\mathrm{FU}$, p-ERK did markedly decrease with the downregulation of Foxc2. By contrast, the levels of p-JNK increased, indicating Foxc2-mediated activation may occur via the MAPK pathway.

\section{Discussion}

Previous studies have demonstrated that Foxc2 is vital in tumor metastasis and metabolism (21). The present study revealed that the development of CRC is associated with a high expression of Foxc2, and a downregulation of Foxc2 enhanced the apoptotic rate of CRC cells. These findings indicate that Foxc2 may act as a potential diagnostic marker in CRC, and a specific inhibitor of Foxc2 may be a novel strategy for the treatment of CRC patients.

Resisting the apoptosis of cells is a hallmark of the majority of cancers (22). At present, the elimination of the resistance to apoptosis is an anticancer therapy (23). Activation of apoptotic 


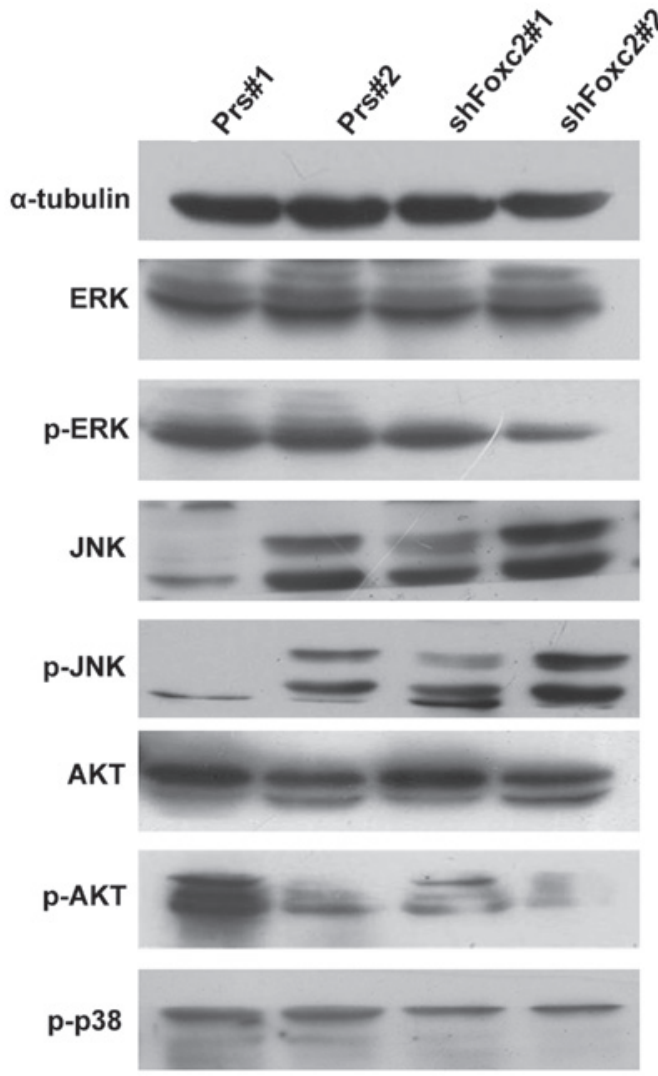

Figure 5. Foxc2 activates the mitogen-activated protein kinase and phosphatidylinositide 3-kinases/protein kinase B signaling pathways. The protein levels of the indicated proteins were determined by western blot analysis. Foxc2, forkhead box C2 gene; 5-FU, 5-fluorouracil; ERK, extracellular-signal-regulated kinases; p, phosphorylated; JNK, c-Jun N-terminal kinases; AKT, protein kinase B; Prs\#1, human colon cancer HCT116 cells without treatment of 5-FU; Prs\#2, human colon cancer HCT116 cells with $2 \mathrm{mg} / 1$ 5-FU; shFoxc2\#1, Foxc2 short hairpin RNA stable cell lines withou treatment of 5-FU; shFoxc2\#2, Foxc2 short hairpin RNA stable cell lines with $2 \mathrm{mg} / 15$-FU.

pathways is currently considered a vital step in the development of tumors $(24,25)$. Therefore, the identification of the mechanism underlying the apoptotic pathway is important. Common stresses inducing apoptosis are imbalances in signaling pathways, resulting in altered levels of oncogene signaling. Alternatively, tumors may increase the expression of anti-apoptotic regulators, including $\mathrm{Bcl}-2$ and $\mathrm{Bcl}-$ extra large, and survival signals, such as insulin-like growth factor 1, by downregulating pro-apoptotic factors, including Bax, Bcl-2 and cleaved-caspase-3, or by eluding the extrinsic ligand-induced death pathway (26).

The present study demonstrated that Foxc2 is vital in creating an anti-apoptotic environment in CRC cells that is relatively insensitive to chemotherapy. The increased expression of Foxc2 in CRC cells enhances the resistance to apoptosis induced by $5-\mathrm{FU}$, a common chemotherapeutic drug used in alimentary canal neoplasm. By contrast, a knockdown of Foxc2 markedly enhanced the sensitivity of the cells to 5-FU; therefore, allowing the cells to undergo apoptosis. The present study concludes that the activity of Foxc2 is associated with the increased survival rate of CRC cells, when 5-FU is used for therapy. Therefore, Foxc2 may be a potential target for chemotherapy.
MAPK and PI3K/AKT signaling pathways are frequently involved in the promotion of proliferation of cells $(20,27,28)$, while inhibition of the MAPK pathway suppresses the proliferation of cells by induction of cell apoptosis through caspase activation (29). The activation of several proteins, including ERK, JNK and AKT, is regulated by the MARK and PI3K/AKT pathways (30-32). The present study investigated whether PI3K and MAPKs are critical in the Foxc2 inhibition of apoptosis, and demonstrated that several MAPK-regulated proteins were upregulated in Foxc2-overexpressing CRC cells and downregulated in Foxc2-inhibited CRC cells. Since Foxc2 is a transcriptional gene, it may not affect the MAPK and PI3K/AKT pathways through phosphorylation directly, and it is evident that the molecular mechanism of how Foxc2 affected the pathway requires additional investigation. Furthermore, several other questions require resolution; the potential role of Foxc2 in CRC cells remains unclear, and the involvement of other signaling pathways in the anti-apoptosis of CRC should be determined, although the present study doubts the involvment of additional pathways. Consequently, additional details concerning the function of Foxc2 require investigation.

In conclusion, the present study demonstrates the methods behind the combination of Foxc2 depletion and 5-FU treatment. A knockdown of Foxc2 induces cancer cells to become more sensitive to 5-FU, and a depletion of Foxc2 enhances 5-FU-induced apoptosis. Furthermore, the present study demonstrated that the MAPK and PI3K/AKT pathways are critical for the development of Foxc2. In the present study, a knockdown of Foxc2 inhibits AKT activation and regulates the expression of Bcl-2 and Bax. In addition, p-ERK markedly decreased with the downregulation of Foxc2 under the treatment with 5-FU. Overall, the present study reveals that a combination of Foxc2 depletion and 5-FU treatment may be a potential clinical therapy for CRC.

\section{Acknowledgements}

The present study was supported by the National Natural Science Foundation of China (grant nos. 30901791, 81172055 and 81071735), Major Projects of the National Natural Science Foundation of China (grant no. 81090422), State Key Program of the National Natural Science Foundation of China (grant no. U1201226), National Basic Research Program of China (The 973 Program; grant nos. 2010 CB529402 and 2010CB529403), Guangdong Provincial Natural Science Foundation of China (grant no. S2012010009643), Zhu Jiang Science and Technology New Star Foundation in Guangzhou City (grant nos. 201212200052 and 2012J2200044), Science and Technology Innovation Foundation of Guangdong Higher Education (grant no. CXZD1016) and Key Program of the National Natural Science Foundation of Guangdong (grant no. 2010B031500012).

\section{References}

1. Siegel R, Naishadham D and Jemal A: Cancer statistics, 2013. CA Cancer J Clin 63: 11-30, 2013.

2. Hendon SE and DiPalma JA: U.S. practices for colon cancer screening. Keio J Med 54: 179-183, 2005.

3. Miura N, Wanaka A, Tohyama M and Tanaka K: MFH-1, a new member of the fork head domain family, is expressed in developing mesenchyme. FEBS Lett 326: 171-176, 1993. 
4. Nishida N, Mimori K, Yokobori T, Sudo T, Tanaka F, Shibata K, Ishii $\mathrm{H}$, Doki $\mathrm{Y}$ and Mori M: FOXC2 is a novel prognostic factor in human esophageal squamous cell carcinoma. Ann Surg Oncol 18: 535-542, 2011

5. Hollier BG, Tinnirello AA, Werden SJ, Evans KW, Taube JH, Sarkar TR, Sphyris N, Shariati M, Kumar SV, Battula VL, et al: FOXC2 expression links epithelial-mesenchymal transition and stem cell properties in breast cancer. Cancer Res 73: 1981-1992, 2013.

6. van der Heul-Nieuwenhuijsen L, Dits NF and Jenster G: Gene expression of forkhead transcription factors in the normal and diseased human prostate. BJU Int 103: 1574-1580, 2009.

7. Papanicolaou KN, Izumiya $Y$ and Walsh K: Forkhead transcription factors and cardiovascular biology. Circ Res 102: 16-31, 2008.

8. Sano H, Leboeuf JP, Novitskiy SV, Seo S, Zaja-Milatovic S, Dikov MM and Kume T: The Foxc2 transcription factor regulates tumor angiogenesis. Biochem Biophys Res Commun 392: 201-206, 2010.

9. Kume T: Foxc2 transcription factor: A newly described regulator of angiogenesis. Trends Cardiovasc Med 18: 224-228, 2008.

10. Watanabe T, Kobunai T, Yamamoto Y, Matsuda K, Ishihara S, Nozawa K, Iinuma H, Kanazawa T, Tanaka T, Konishi T, et al: Gene expression of mesenchyme forkhead 1 (FOXC2) significantly correlates with the degree of lymph node metastasis in colorectal cancer. Int Surg 96: 207-216, 2011.

11. Subramanya RD, Coda AB and Sinha AA: Transcriptional profiling in alopecia areata defines immune and cell cycle control related genes within disease-specific signatures. Genomics 96 146-153, 2010

12. Petrova TV, Karpanen T, Norrmén C, Mellor R, Tamakoshi T, Finegold D, Ferrell R, Kerjaschki D, Mortimer P, Ylä-Herttuala S, et al: Defective valves and abnormal mural cell recruitment underlie lymphatic vascular failure in lymphedema distichiasis. Nat Med 10: 974-981, 2004

13. Cui YM, Jiang D, Zhang SH, Wu P, Ye YP, Chen CM, Tang N, Liang L, Li TT, Qi L, et al: FOXC2 promotes colorectal cancer proliferation through inhibition of FOXO3a and activation of MAPK and AKT signaling pathways. Cancer Lett 353: 87-94, 2014

14. Likhite $\mathrm{N}$ and Warawdekar UM: A unique method for isolation and solubilization of proteins after extraction of RNA from tumor tissue using trizol. J Biomol Tech 22: 37-44, 2011.

15. Mani SA, Yang J, Brooks M, Schwaninger G, Zhou A, Miura N, Kutok JL, Hartwell K, Richardson AL and Weinberg RA: Mesenchyme Forkhead 1 (FOXC2) plays a key role in metastasis and is associated with aggressive basal-like breast cancers. Proc Natl Acad Sci USA 104: 10069-10074, 2007.

16. Hahn WC, Dessain SK, Brooks MW, King JE, Elenbaas B, Sabatini DM, DeCaprio JA and Weinberg RA: Enumeration of the simian virus 40 early region elements necessary for human cell transformation. Mol Cell Biol 22: 2111-2123, 2002.

17. Liao WT, Wang X, Xu LH, Kong QL, Yu CP, Li MZ, Shi L, Zeng MS and Song LB: Centromere protein $\mathrm{H}$ is a novel prognostic marker for human nonsmall cell lung cancer progression and overall patient survival. Cancer 115: 1507-1517, 2009.
18. Livak KJ and Schmittgen TD: Analysis of relative gene expression data using real-time quantitative PCR and the $2^{-\Delta \Delta C T}$ Method. Methods 25: 402-408, 2001.

19. Fresno Vara JA, Casado E, de Castro J, Cejas P, Belda-Iniesta C and González-Barón M: PI3K/Akt signalling pathway and cancer. Cancer Treat Rev 30: 193-204, 2004.

20. Massagué J: G1 cell-cycle control and cancer. Nature 432: 298-306, 2004

21. Watanabe A, Suzuki H, Yokobori T, Altan B, Kubo N, Araki K, Wada S, Mochida Y, Sasaki S, Kashiwabara K, et al: Forkhead box protein $\mathrm{C} 2$ contributes to invasion and metastasis of extrahepatic cholangiocarcinoma, resulting in a poor prognosis. Cancer Sci 104: 1427-1432, 2013.

22. Hanahan D and Weinberg RA: Hallmarks of cancer: The next generation. Cell 144: 646-674, 2011

23. Liu JJ, Lin M, Yu JY, Liu B and Bao JK: Targeting apoptotic and autophagic pathways for cancer therapeutics. Cancer Lett 300: 105-114, 2011.

24. Denicourt C and Dowdy SF: Medicine. Targeting apoptotic pathways in cancer cells. Science 305: 1411-1413, 2004.

25. Hougardy BM, Maduro JH, van der Zee AG, Willemse PH, de Jong S and de Vries EG: Clinical potential of inhibitors of survival pathways and activators of apoptotic pathways in treatment of cervical cancer: Changing the apoptotic balance. Lancet Oncol 6: 589-598, 2005.

26. Kang MH and Reynolds CP: Bcl-2 inhibitors: Targeting mitochondrial apoptotic pathways in cancer therapy. Clin Cancer Res 15: 1126-1132, 2009.

27. Rinn JL, Wang JK, Allen N, Brugmann SA, Mikels AJ, Liu H, Ridky TW, Stadler HS, Nusse R, Helms JA and Chang HY: A dermal HOX transcriptional program regulates site-specific epidermal fate. Genes Dev 22: 303-307, 2008.

28. Leonis MA, Thobe MN and Waltz SE: Ron-receptor tyrosine kinase in tumorigenesis and metastasis. Future Oncol 3: 441-448, 2007.

29. Tsuchiya T, Tsuno NH, Asakage M, Yamada J, Yoneyama S, Okaji Y, Sasaki S, Kitayama J, Osada T, Takahashi K and Nagawa H: Apoptosis induction by p38 MAPK inhibitor in human colon cancer cells. Hepatogastroenterology 55: 930-935, 2008.

30. Mao JD, Wu P, Huang JX, Wu J and Yang G: Role of ERK-MAPK signaling pathway in pentagastrin-regulated growth of large intestinal carcinoma. World J Gastroenterol 20: 12542-12550, 2014.

31. Esmaeili MA, Farimani MM and Kiaei M: Anticancer effect of calycopterin via PI3K/Akt and MAPK signaling pathways, ROS-mediated pathway and mitochondrial dysfunction in hepatoblastoma cancer (HepG2) cells. Mol Cell Biochem 397: 17-31, 2014.

32. Chen JY, Zhang L, Zhang H, Su L and Qin LP: Triggering of p38 MAPK and JNK signaling is important for oleanolic acid-induced apoptosis via the mitochondrial death pathway in hypertrophic scar fibroblasts. Phytother Res 28: 1468-1478, 2014. 